\title{
Quantum theory for the nanoscale propagation of light through stacked thin film layers
}

\author{
Kayn A. Forbes, Mathew D. Williams and David L. Andrews* \\ School of Chemistry, University of East Anglia, Norwich NR4 7TJ, United Kingdom
}

\begin{abstract}
Stacked multi-layer films have a range of well-known applications as optical elements. The various types of theory commonly used to describe optical propagation through such structures rarely take account of the quantum nature of light, though phenomena such as Anderson localization can be proven to occur under suitable conditions. In recent and ongoing work based on quantum electrodynamics, it has been shown possible to rigorously reformulate, in photonic terms, the fundamental mechanisms that are involved in reflection and optical transmission through stacked nanolayers. Accounting for sum-over-pathway features in the quantum mechanical description, this theory treats the sequential interactions of photons with material boundaries in terms of individual scattering events. The study entertains an arbitrary number of reflections in systems comprising two or three internally reflective surfaces. Analytical results are secured, without recourse to FTDT (finite-difference time-domain) software or any other finite-element approximations. Quantum interference effects can be readily identified. The new results, which cast the optical characteristics of such structures in terms of simple, constituent-determined properties, are illustrated by model calculations.
\end{abstract}

Keywords: Thin films, photonic crystals, surface reflection, distributed Bragg reflectors, photonics, quantum electrodynamics, quantum optics.

\section{INTRODUCTION}

The field of thin film optics ${ }^{1}$ deals with layers of material with thicknesses typically in the range of nanometers to several micrometers. There are many different examples of material used to create thin films including those of both dielectric ${ }^{2}$ and metallic character. ${ }^{3}$ Well-known examples of their use include anti-reflective ${ }^{4}$ and high-reflection coatings ${ }^{5}$, with more contemporary applications including drug delivery, ${ }^{6}$ electronics, ${ }^{7}$ and solar energy systems. ${ }^{8}$ The simplest effect of thin films in conjunction with light can be understood through what is known as thin film interference, where the throughput is modified through transmission and reflection. This optical thin film theory can be regarded as Maxwell theory applied to electromagnetic waves that are propagating across layered systems, where the thickness of the layers is of the order of the wavelength of the light. For films that are stacked in layers separated by nanoscale dimensions, i.e. much less than an optical wavelength, other multiple-reflection effects come more prominently into play. Moreover, at low levels of intensity, quantum effects can be anticipated. To investigate the optical behavior in the interactions between films and light in this regime invites the use of quantum electrodynamics - QED, ${ }^{9}$ the theory in which due heed is paid to the quantum nature of both matter and radiation. The application of these methods offers a route to understand more fully the nature of the physical mechanisms involved in the multiple reflections of a single photon.

\section{QUANTUM THEORY OF LIGHT REFLECTION AND PROPAGATION}

We begin by introducing a suitable quantum framework to describe the propagation of off-resonant light through multifaceted media that are partially transmissive and partially reflective. These are media, in other words, in which each

Nanophotonics VI, edited by David L. Andrews, Jean-Michel Nunzi, Andreas Ostendorf, Proc. of SPIE Vol. 9884, 988434 · C 2016 SPIE · CCC code: 0277-786X/16/\$18 · doi: 10.1117/12.2227694 
incident photon emerges travelling either in the same direction as the input, or in some other direction. After developing the basic theory, we shall then restrict attention to cases where scattering events are limited to those associated with a series of internal, parallel surfaces, generating specular retro-reflection.

A suitable starting point is an equation for an observable that signifies the rate at which photons from a given input emerge in an identifiable direction. This rate can be developed from the corresponding quantum amplitude, connecting a given initial and final radiation state, using the Fermi rule. For elastic processes that leave the material system unchanged, each of the quantum amplitudes $M_{f i}^{(\xi)}$, relating to interactions with one of $N$ discrete optical centers labelled $\xi$, have to be added (since their effects cannot be experimentally differentiated). Separating single-center and interference terms, most conveniently cast together in the form of a Fermi Rule, the rate is accordingly expressible in the following form:

$$
\Gamma=\frac{2 \pi}{\hbar} \rho_{f}\left[\sum_{\xi}^{N}\left\langle\left|M_{f i}^{(\xi)}\right|^{2}\right\rangle+\sum_{\xi^{\prime} \neq \xi}^{N} \sum_{\xi}^{N}\left\langle M_{f i}^{(\xi)}\right\rangle\left\langle\bar{M}_{f i}^{\left(\xi^{\prime}\right)}\right\rangle\right]
$$

in which $\rho_{f}$ is a density of final radiation states (relating to a small angular spread around the direction of scattering), and where angular brackets denote a distributional (i.e. both translational and orientational) average. Now for any form of scattering we can identify a difference in the wave-vectors for the photon input and output modes, defined as $\Delta \mathbf{k}$ (which will be zero in the singular case of forward scattering). By the application of second order time-dependent perturbation theory it is readily shown ${ }^{9}$ that the quantum amplitude for a molecule $\xi$ takes the form

$$
M_{f i}^{(\xi)} \sim \alpha_{i j}^{\xi}(\omega) \bar{e}_{i} e_{j}^{\prime} e^{i \Delta \mathbf{k} \cdot \mathbf{R}_{\xi}}
$$

where the tensor components $\alpha_{i j}^{\xi}(\omega)$ represent the molecular property known as the dynamic polarizability, $e_{i}$ represents a (Cartesian) component of the polarization vector for the input electric field, its primed counterpart the scattered output, with an overbar denoting complex conjugation, and where $\mathbf{R}_{\xi}$ is the position coordinate of center $\xi$. Since the quantum amplitude carries a phase factor $\mathrm{e}^{i \Delta \mathbf{k} \cdot \mathbf{R}_{\xi}}$, then assuming there is no positional or orientational correlation between different scatterers, (a condition that will apply to any disordered solid or fluid), use of the ergodic theorem enables equation (1.1) to be rewritten as

$$
\begin{gathered}
\Gamma=\frac{2 \pi}{\hbar} \rho_{f}\left[N\left\langle\left|M_{f i}^{(\xi)}\right|^{2}\right\rangle+\left(\eta_{N}-N\right)\left|\left\langle M_{f i}^{(\xi)}\right\rangle\right|^{2}\right], \\
\eta_{N}=\left|\sum_{\xi}^{N} \mathrm{e}^{i \Delta \mathbf{k} \cdot \mathbf{R}_{\xi}}\right|^{2} .
\end{gathered}
$$

Here, the factor $\eta_{N}$ may be termed a coherence factor. The first term in square brackets of (1.3) thus involves the polarizability quadratically, as an outer product tensor of rank 4; there are four polarization vector components that also combine to produce a field tensor of the same rank. Due to the linear dependence of this term on the number of scatterers, the contribution it represents from independent centers is said to be incoherent. Physically this means that the scattering amplitudes for the optical centers in a sample interfere randomly, and the intensity is therefore a sum of $N$ equivalent scattering rates. Non-forward Rayleigh scattering and diffusive reflection are therefore both examples of incoherent scattering processes. The second term of (1.3) by contrast involves a square of the inner product of rank 2 polarizability and polarization component tensors. Its contribution is termed coherent due to the scattered intensity exhibiting a quadratic dependence on the number of optical centers, in contrast to the linear dependence of $N$ in incoherent scattering. However, as will be shown, this coherent term can only persist in special conditions. 
At this juncture we have to specify the physical property of a surface, comprising the molecules $\xi$, which can allow forward transmission or give rise to specular reflection; we develop this from an original foundation in theory due to Atkins and Barron. ${ }^{10}$ It is to be assumed that these molecules constitute a planar surface - and for simplicity we shall also assume that the input light impinges upon it at a normal angle of incidence. The corresponding local polarizability is effectively the value that corresponds to the trans-interface change in refractive index, as determined by the Lorentz-Lorenz formula. Bulk regions of solid through which the light passes lead only to a net destructive interference - unless $\Delta \mathbf{k}=0$, signifying onward (forward) propagation. At surfaces and interfaces, a distinction now arises between the parametric processes of forward and retro-scattering: the transmission of light again invokes the condition for a coherent process through wavevector matching $\Delta \mathbf{k}=0$, whilst specular reflection fulfils the criteria through the relation $\mathbf{R}_{\xi} \perp \Delta \mathbf{k}=0$. The matrix amplitudes for both transmission and specular reflection are therefore independent on the position of each scatterer, and the amplitudes from different optical centers interfere constructively. Again, the reasonable assumption that molecular displacement vectors $\mathbf{R}_{\xi}$ possess a completely random spatial distribution leads to the following result, ${ }^{11}$

$$
\eta_{N} \approx N^{2}(\Delta \mathbf{k}=0)
$$

\section{LIGHT PROPAGATION THROUGH THIN FILMS}

We now consider a model system in which light passes through a series of three parallel, partially reflective surfaces. This is a system that exhibits some notable features of number theory; ${ }^{12-13}$ our interest lies in the physical mechanisms that forge the optical properties of such a system. In principal the surfaces might be three atomically thin layers such as graphene, separated by a vacuum; the same model can also represent a pair of two stacked thin films of a different composition, such that a difference in their refractive indices represents a dielectric discontinuity. We lean to the latter interpretation: however, it is worth noting that the general methods we shall use are applicable to an arbitrary-sized stack of thin films. The system to be studied is shown schematically in Figure 1, illustrating the fact that a photon entering the system can then propagate through it with an arbitrary number of internal reflections before being emitted from the thin film stack and sequentially annihilated at a detector.

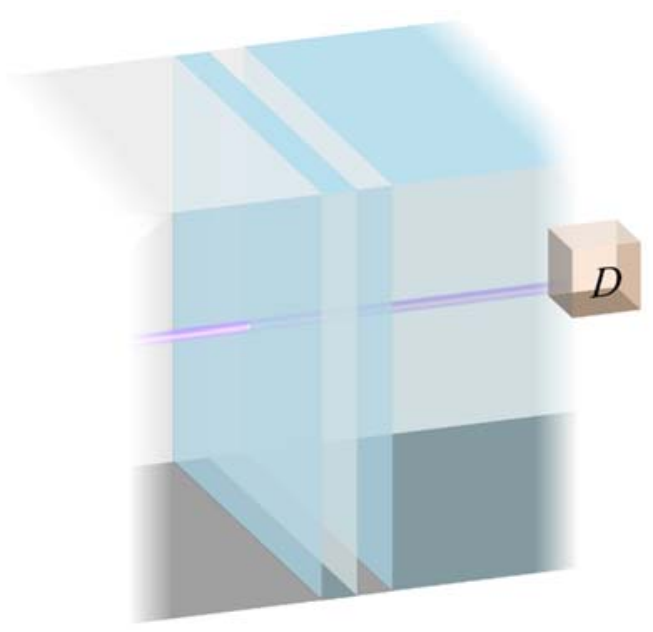

Figure 1. Schematic depiction of light propagating through an optical system that comprises three parallel interfaces, each separating two regions of material with differing refractive index. The light is incident from a source on the left, and the observed transmission is measured by a detector D shown on the right. 
We shall denote each specific contribution to the output electric field, as registered by a remote (far-field) detector on the opposite side from the source, as $\mathscr{C}_{(m, n) i}$, where $m$ registers the accumulated path delay and $n$ represents the number of internal reflections. The simplest case is a dynamic electric field free from interactions with the system it traverses, for which $C_{m, n) i}^{\prime}$ is given by:

$$
\mathscr{C}_{(0,0) i}^{\prime}=e_{i} \mathrm{e}^{i \mathbf{k} \cdot \mathbf{R}}
$$

For present purposes, in which we assume propagation in a direction that is normal to each surface, the scalar product in the phase exponential can be written as simply $k R$. We shall specify the effective polarizability per unit interfacial volume as $\alpha_{i j}^{(n)}(\omega)$, where the superscript integer signifies one of the three specific interfaces.

In principle, by deploying a quantum electrodynamical representation, each successive transit of a 'reflected' photon to its next interface encounter should rather be regarded as the propagation of a virtual photon, since no intermediary optical mode is observable in either the initial nor the final state of the system. ${ }^{14-15}$ (Note, here the ultimate transmitted light detection process is regarded as the interaction that leads the system into its final state.) Formulating each transit in such terms, in which all the virtual photon has a wave-vector contributions of unrestricted directions and magnitudes, leads to a resonance dipole-dipole interaction tensor exhibiting retardation features - and in fact entirely consistent with the result of retarded coupling that emerges from classical electrodynamical theory. ${ }^{16}$ These features are responsible for an evolution over distance $R$ from an $R^{-3}$ dependence in the short-range to $R^{-1}$ in the wave-zone. The explicit form of the wellknown index-symmetric virtual photon coupling tensor, $V_{i j}(k, \mathbf{R})$, which serves as a Green's function and itself carries a phase factor $e^{i k R}$, is given explicitly elsewhere. ${ }^{17}$ However, it has been shown in other recent work that propagation over nanoscale dimensions, between structures that determine an axial confinement, are better represented by a Green's function that suffers no diminution with distance; for present purposes we shall label this as $W_{i j}$.

In the context of describing multiple reflections, another important feature of the quantum electrodynamical representation is accommodating a sum over all possible pathways for any particular process, with even the physically non-intuitive contributions taken into account for the overall amplitude. It transpires that the number of possible ways a photon can be transmitted or reflected within a thin film stack invokes time-orderings whose number relates to the Fibonacci series. For an odd number of reflections within the film stack, the overall outcome for the incident photon is for it to be reflected back towards the source i.e. a photon momentum of $(-\mathbf{k})$. When a photon is reflected within the stack an even number of times, it is seen to progress with the same wave-vector from which it left the source $(\mathbf{k})$ and its direction is towards that of the detector; thus it is manifest as transmitted through the thin film layers. Figure 2 illustrates the various transmission routes that entail specifically four internal reflections.

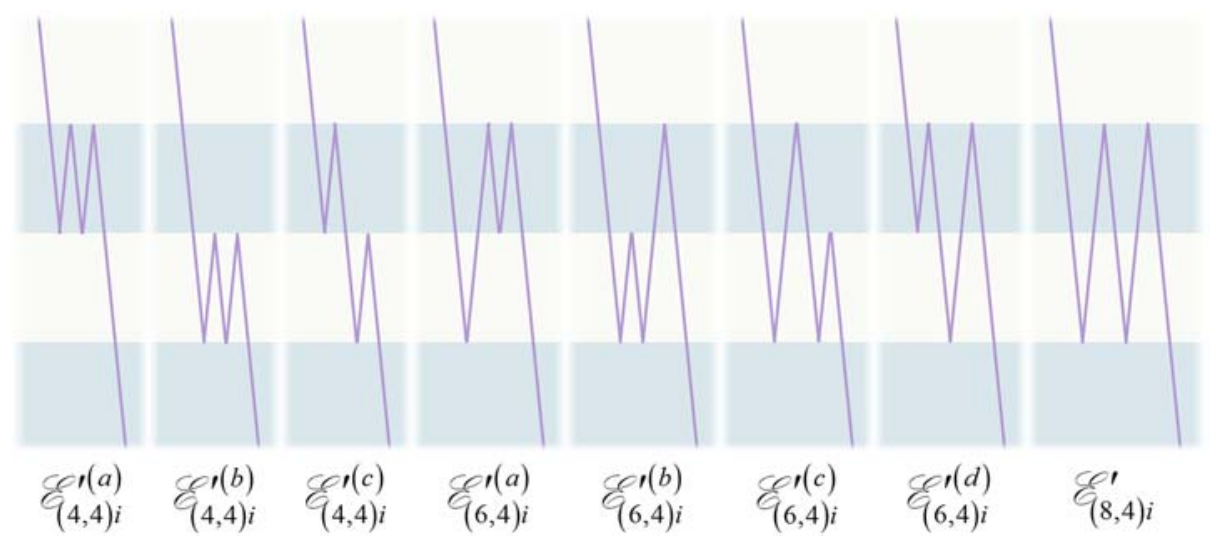

Figure 2. Transmission routes (top to bottom) with four internal reflections, shown skewed from the normal for clarity. 
It is instructive to work through a particular pathway that contributes to the transmittance of a photon through a pair of thin films stacked against one another. Take, for example, one of the amplitudes for the electric field output, $\mathscr{C}_{(6,4) i}^{\prime}$, where there is a relative path delay of 6 layer spacings, (compared to that of the photon being transmitted without any interaction with matter), and the light undergoes 4 reflections within the two thin films. The pathway is represented in Figure 3, where for clarity the lines that represent the photon pathway are offset. It is worth noting that the diagram is effectively a multicenter Feynman diagram, in which we can use a two-photon collapsed vertex formulation ${ }^{18}$ for each reflection. The logic for this is simply that the interval between successive reflections at different surfaces is significantly longer than the immeasurably short timescale over which the photon annihilation and creation together comprise each surface scattering event. Taking it step by step: the photon from the source first propagates to interface 3, where it suffers annihilation. From the same interface a virtual photon is created and couples with interface 1, via the coupling tensor $W_{p o}$.
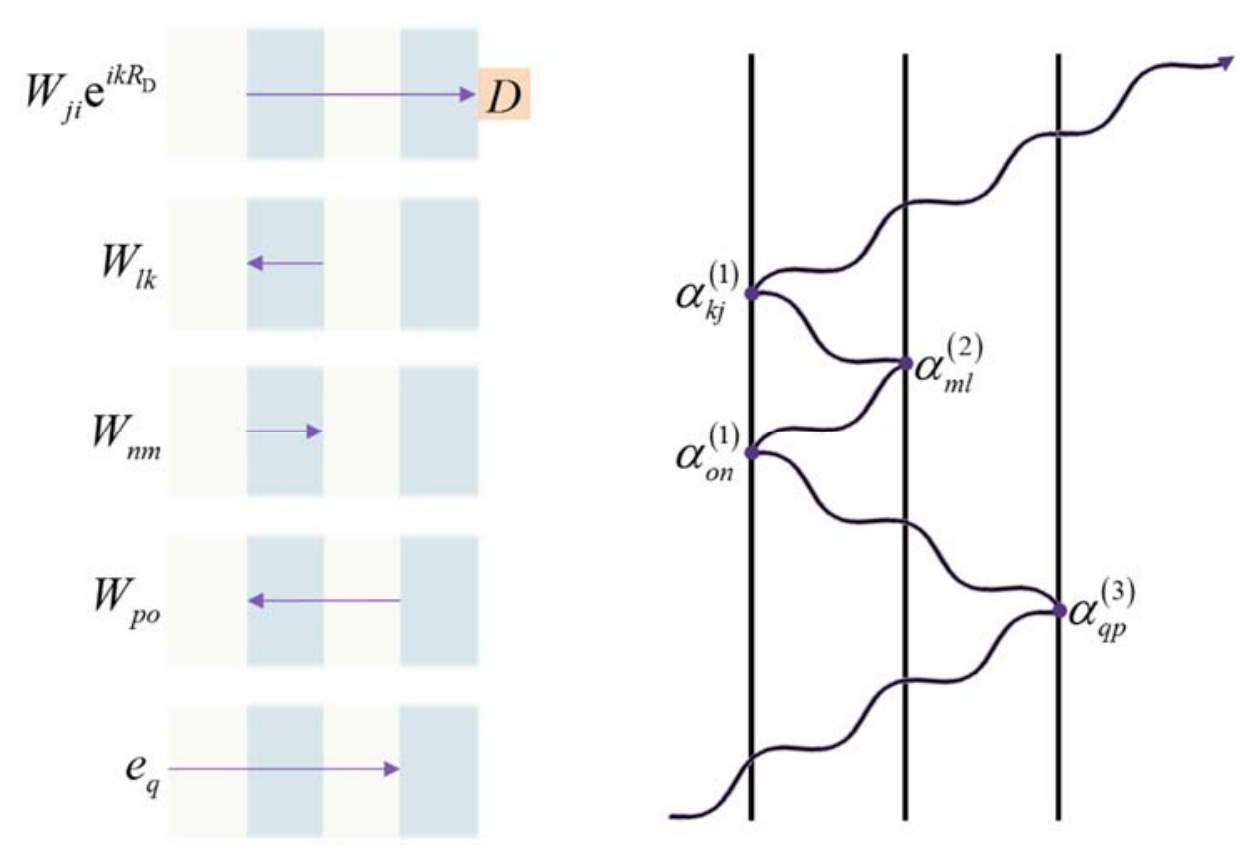

Figure 3. One of eight distinct pathways that sum to give the quantum amplitude for light scattering through two thin films undergoing four internal reflections. Lines are offset from normal incidence for clarity.

A further two couplings occur between interfaces 1 and 2, until the final coupling to a remote detector takes place through the propagator $W_{j i} \mathrm{e}^{i k R_{D}}$. The overall electric field output for this particular path, i.e. one term in the overall quantum amplitude for transmission, can be written as,

$$
C_{(6,4) i}^{\prime}=e_{q} \alpha_{q p}^{(3)}(\omega) W_{p o} \alpha_{o n}^{(1)}(\omega) W_{n m} \alpha_{m l}^{(2)}(\omega) W_{l k} \alpha_{k j}^{(1)}(\omega) W_{j i} \mathrm{e}^{i k R_{D}}
$$

To proceed further, we make a simplifying assumption, namely that the films are of significantly sub-wavelength thickness, i.e. sufficiently thin that the phase delay associated with different numbers of internal reflections need not be taken into account. Moreover, consistent with this level of approximation, the distance dependence of the propagation tensor elements can also be suppressed. Lastly, we shall assume that the effective local polarizability responsible for each reflection has the same value - as indeed can apply to a system comprising films of alternating composition (i.e. presenting surfaces of a constant difference in magnitude between the interfacing materials). Then, by inspection of the specific form 
of the above result, it is evident that the quantum amplitude for an arbitrary number of reflections $p$ can be concisely expressed in the following form;

$$
\mathscr{C}_{(p) i_{0}}^{\prime}=F_{p+2} e_{i_{2 p}} \prod_{j=0}^{p-1} \alpha_{i_{i_{j}+2} i_{2 j+1}} W_{i_{2 j+1} i_{2 j}}
$$

In this expression, the multiplicand in fact expresses a reflection amplitude conventionally represented as $r$. For transmission - accommodating an arbitrary (even) number of individual reflections, the overall quantum amplitude comprises a sum of such terms, i.e.

$$
C_{i_{0}}^{\prime}=\sum_{n=0}^{\infty} C_{(2 n) i_{0}}^{\prime}=e_{\text {in }} \sum_{n=0}^{\infty} F_{2 n+2} r^{2 n}
$$

This falls in the form of a series $g\left(r^{2}\right)$ whose even powers of $r^{2}$ carry coefficients given by the alternating terms in the Fibonacci series - see Table 1.

$$
g\left(r^{2}\right)=1+3 r^{2}+8 r^{4}+21 r^{6}+55 r^{8}+144 r^{10}+\ldots
$$

The analysis of this series reveals that, within a radius of convergence $r<0.618$, i.e. the inverse of the Golden Ratio, the result emerges as,

$$
g\left(r^{2}\right)=\frac{1}{1-3 r^{2}+r^{4}}
$$

From this, we can evaluate the intensity of transmitted light as taking the form $I_{\mathrm{t}} \sim g^{2}\left(r^{2}\right)$,

$$
I_{\mathrm{t}} \sim\left(\frac{1}{1-3 r^{2}+r^{4}}\right)^{2}
$$

By similar arguments the net intensity of retro-reflected light (i.e. a sum of all odd-numbering reflections) follows as,

$$
I_{\mathrm{r}} \sim\left(\frac{r^{2}\left(3-4 r^{2}+r^{4}\right)}{1-3 r^{2}+r^{4}}\right)^{2}
$$

It is then a straightforward matter to assess the variation in fractional optical throughput with the coefficient of surface reflection, $R=r^{2}$. In figure 4 we plot the results for $I_{\mathrm{t}} /\left(I_{\mathrm{t}}+I_{\mathrm{r}}\right)$ over the physically meaningful convergence range for the amplitude measure of reflectivity. The most notable feature is the minimum, signifying a corresponding maximum in the overall system reflectivity.

Table 1. The Fibonacci numbers associated with counting internal reflections

\begin{tabular}{l|l|l|l|l|l|l} 
Number of Pathways $\left(F_{p+2}\right)$ & 1 & 3 & 8 & 21 & 55 & 144 \\
\hline Number of Reflections $(p)$ & 0 & 2 & 4 & 6 & 8 & 10
\end{tabular}




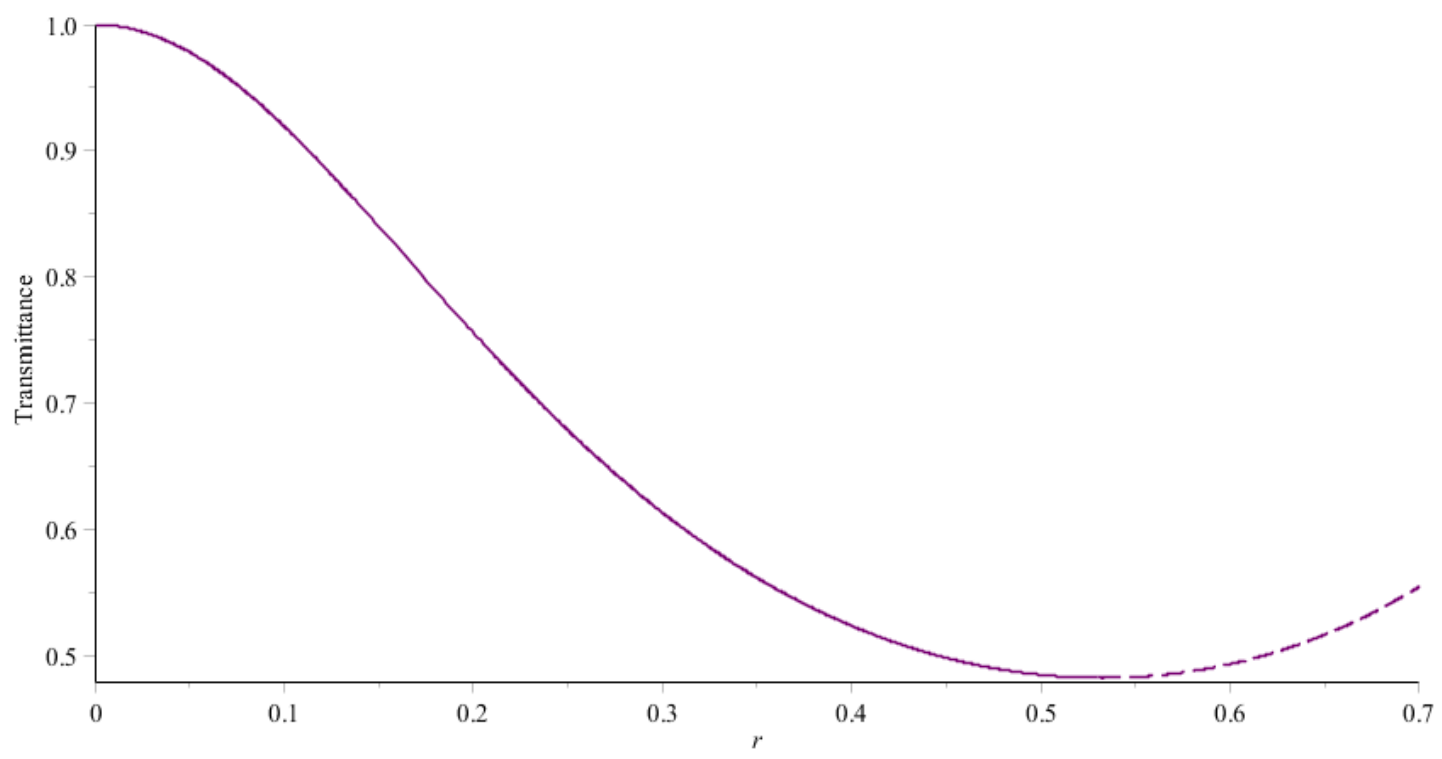

Figure 4. Variation in system transmission as a function of the effective amplitude reflectivity $r$.

\section{DISCUSSION}

The presented analysis of a stacked-film system at a fundamental, quantum electrodynamical level elicits the physical mechanisms operating at several tiers of optical interaction, including individual photon 'reflections', the role of constructive interference at surfaces and interfaces, and the interplay of reflection sequences. The model we have deployed to derive these first, preliminary results has been designed to exhibit the formalism. It relates to real but simple optical configurations; nonetheless the power of the methods we have used opens the ground for a detailed analysis and a determination of optical properties for wide-ranging multi-surface systems. The present, simplifying assumptions and approximations have served only to enable analytical results to be secured, and to simplify the presentation. It is worth emphasizing that none of them is pivotal to this form of analysis, nor is there any need for recourse to FTDT (finitedifference time-domain) software or any other finite-element approximations.

Dealing with the evolution of the optical state in the course of individual photon throughput has enabled quantum amplitudes to be derived, such that the derived signal intensity as registered by a detector properly accommodates the interplay of different sequences of internal reflection. Moreover, tackling the physics at this level provides equations from which specific quantum interference effects can also be readily identified. In further developments the theory can now be applied in calculations on a variety of technically significant optical elements. In consequence it will be possible to identify the precise effects of the stack structures in determining, for a given optical input, not only overall the system transmissivity as a function of material properties and geometry, but also the extent of features such as pulse broadening and photon delay time.

\section{ACKNOWLEDGEMENTS}

The authors would like to thank the University of East Anglia for funding this research. 


\section{REFERENCES}

[1] Knittl, Z., [Optics of thin films: an optical multilayer theory], Wiley, London (1976).

[2] Scott, J. F., "High-dielectric constant thin films for dynamic random access memories (DRAM)," Annu. Rev. Mater. Sci. 28, 79-100 (1998).

[3] Abdulhalim, I., "Plasmonic sensing using metallic nano-sculptured thin films," Small 10, 3499-3514 (2014).

[4] Raut, H. K., Ganesh, V. A., Nair, A. S. and Ramakrishna, S., "Anti-reflective coatings: A critical, in-depth review," Energy Environ. Sci. 4, 3779-3804 (2011).

[5] Soderstrom, K., Haug, F. J., Escarre, J., Pahud, C., Biron, R. and Ballif, C., "Highly reflective nanotextured sputtered silver back reflector for flexible high-efficiency n-i-p thin-film silicon solar cells," Sol. Energy Mater. Sol. Cells 95, 3585-3591 (2011).

[6] Zelikin, A. N., "Drug releasing polymer thin films: New era of surface-mediated drug delivery," ACS Nano 4, 2494$2509(2010)$.

[7] Eda, G. and Chhowalla, M., "Graphene-based composite thin films for electronics," Nano Lett. 9, 814-818 (2009).

[8] Li, J. S., Yu, H. Y., Wong, S. M., Zhang, G., Sun, X. W., Lo, P. G. Q. and Kwong, D. L., "Si nanopillar array optimization on Si thin films for solar energy harvesting," Appl. Phys. Lett. 95, 033102 (2009).

[9] Craig, D. P. and Thirunamachandran, T., [Molecular Quantum Electrodynamics: An Introduction to RadiationMolecule Interactions], Dover Publications, Mineola, NY (1998).

[10] Atkins, P. W. and Barron, L. D., "Rayleigh scattering of polarized photons by molecules," Mol. Phys. 16, 453-466 (1969).

[11] Marcuse, D., [Principles of quantum electronics], Elsevier, NY (2012).

[12] Junge, B. and Hoggatt Jr, V., "Polynomials Arising from Reflections Across Multiple-Plates," Fibonacci Quarterly 11, 285-291 (1973).

[13] Grimaldi, R., [Fibonacci and Catalan Numbers: an introduction], John Wiley \& Sons, New Jersey (2012).

[14] Andrews, D. L. and Bradshaw, D. S., "Virtual photons, dipole fields and energy transfer: a quantum electrodynamical approach," Eur. J. Phys. 25, 845-858 (2004).

[15] Salam, A., [Molecular Quantum Electrodynamics. Long-Range Intermolecular Interactions], Wiley, Hoboken, NJ (2010).

[16] Jackson, J. D., [Classical Electrodynamics], Wiley, New York (1998).

[17] Daniels, G. J., Jenkins, R. D., Bradshaw, D. S. and Andrews, D. L., "Resonance energy transfer: The unified theory revisited," J. Chem. Phys. 119, 2264-2274 (2003).

[18] Power, E. A. and Thirunamachandran, T., "On the nature of Hamiltonian for interaction of radiation with atoms and molecules: (e/mc)p.A, - $\mu . E$, and all that," Am. J. Phys. 46, 370-378 (1978).

*david.andrews@physics.org 